\title{
Influence of the Core-Valence Interaction and of the Pseudopotential Approximation on the Electron Self-Energy in Semiconductors
}

\author{
Ricardo Gómez-Abal, Xinzheng Li, and Matthias Scheffler \\ Fritz-Haber-Institut der Max-Planck-Gesellschaft, Faradayweg 4-6, D-14195, Berlin, Germany \\ Claudia Ambrosch-Draxl \\ Chair of Atomistic Modeling and Design of Materials, University of Leoben, A-8700, Austria \\ (Received 1 February 2008; published 4 September 2008)
}

\begin{abstract}
State-of-the-art theory addresses single-electron excitations in condensed matter by linking densityfunctional theory (DFT) with many-body perturbation theory. In actual calculations it is common to employ the pseudopotential (PP) approach, where pseudo-wave-functions enter the calculation of the selfenergy, and the core-valence interaction is treated at the DFT level. In this Letter we present accurate allelectron calculations of the self-energy and systematically compare the results to those of PP calculations. The analysis for a range of different materials reveals that both above mentioned approximations are indeed problematic.
\end{abstract}

DOI: 10.1103/PhysRevLett.101.106404

The electron band structure is the central quantity in the characterization of the electronic properties of a solid. It corresponds to the single-electron excitations typically measured by direct or inverse photoemission experiments. The interpretation of the experimental results requires the theoretical understanding of the processes involved. In this aspect, many-body perturbation theory (MBPT) provides the formal basis for evaluating the experimentally observed quasiparticle (qp) band structure. Applying Hedin's $G W$ approximation [1] as a perturbation $\left(G_{0} W_{0}\right)$ to the fictitious, noninteracting Kohn-Sham (KS) electrons of density-functional theory (DFT) [2] provides an accurate description of the electron self-energy. Most $G_{0} W_{0}$ calculations so far employed additional (somewhat hidden) approximations, namely, the replacement of the valence wave functions by pseudo-wave-functions (i.e., a smoothening and the removal of nodes in the atomic-core region) and a linearized DFT treatment of the exchange-correlation (xc) interaction between core and valence electrons. While for ground-state DFT calculations both approximations are well understood and can be controlled, their role for a description of excited states is unclear. Beyond doubt, $G_{0} W_{0}$ pseudopotential (PP) calculations have been impressively successful in reproducing band gaps of semiconductors and insulators [3]. However, first full-potential allelectron (AE) $G_{0} W_{0}$ results [4] have revealed discrepancies, i.e., giving band gaps typically smaller than the PP theory, with a noticeable deviation from the experimental values. Presently it is unclear why the generally more precise $\mathrm{AE} G_{0} W_{0}$ theory often gives worse results than the PP $G_{0} W_{0}$ approach.

In this Letter we will address this question, and, in particular, we will analyze the influence of the two above mentioned approximations of the PP approach. Results for several, distinct crystals, namely, C, Si, BN, AlP, GaAs, $\mathrm{LiF}, \mathrm{NaCl}$, and $\mathrm{CaSe}$ are presented in order to demonstrate the sign and magnitude of the various contributions. We
PACS numbers: 71.15.Qe, 71.10.-w, 71.20.Mq, 71.20.Nr

find that errors in the self-energy and xc potential can be as large as several $\mathrm{eV}$, but because of some favorable cancellation they are reduced to a few tenths of an $\mathrm{eV}$, at least in the systems investigated here. In general, our results reveal that more care is necessary when employing the standard PP approach to excited states, or in other words, safe grounds are only found in all-electron studies.

One criticism [5,6] of earlier AE results [4] was the lack of convergence with respect to the number of excited states included in the calculation of the correlation term of the self-energy. While this was correct in the beginning, recent work [7-9] as well as our results, reported below, reveal that noticeable differences between $\mathrm{AE}$ and PP $G_{0} W_{0}$ calculations do exist, even when the convergence issue has been settled. For example, AE calculations give the fundamental band gap of $\mathrm{Si}$ as $0.95-1.05 \mathrm{eV}[7,8]$ while the PP values range from 1.18 to $1.29 \mathrm{eV}$ [10-13], slightly larger than the experimental counterpart of $1.17 \mathrm{eV}$. For generalized pseudopotentials, where only the $1 s$ shell is treated as core (and hence omitted in the calculation of the self-energy) the band gap reduces to $1.04 \mathrm{eV}$, improving the agreement with $\mathrm{AE}$ data [6].

In $G_{0} W_{0}$ calculations, the qp band structure is obtained from the KS eigenvalues as

$$
\epsilon_{n \mathbf{k}}^{\mathrm{qp}}=\epsilon_{n \mathbf{k}}^{\mathrm{KS}}+\Delta \epsilon_{n \mathbf{k}} .
$$

The correction term, $\Delta \epsilon_{n \mathbf{k}}$, is given by the difference between the diagonal matrix elements of the self-energy $(\Sigma)$ and the KS xc potential $\left(V_{\mathrm{xc}}\right)$

$$
\begin{aligned}
\Delta \epsilon_{n \mathbf{k}}= & \operatorname{Re}\left(\left\langle\phi_{n \mathbf{k}}\left|\sum\left(\left[\left\{\phi_{n \mathbf{k}}, \phi_{\text {core }}\right\}\right], \epsilon_{n \mathbf{k}}^{\mathrm{qp}}\right)\right| \phi_{n \mathbf{k}}\right\rangle\right) \\
& -\left\langle\phi_{n \mathbf{k}}\left|V_{x c}[n]\right| \phi_{n \mathbf{k}}\right\rangle .
\end{aligned}
$$

Here $\Sigma$ depends on all core states, and on all occupied and unoccupied valence states. In the $G_{0} W_{0}$ approximation only the first term in the expansion of $\Sigma$ in powers of the dynamically screened Coulomb potential is included, 


$$
\Sigma\left(\mathbf{r}, \mathbf{r}^{\prime} ; \omega\right)=\frac{i}{2 \pi} \int G_{0}\left(\mathbf{r}, \mathbf{r}^{\prime} ; \omega+\omega^{\prime}\right) W_{0}\left(\mathbf{r}, \mathbf{r}^{\prime} ; \omega^{\prime}\right) d \omega^{\prime},
$$

where $G_{0}$ is the Green function and $W_{0}$ is the screened Coulomb potential, both calculated using the ground-state KS wave functions and eigenvalues.

In the KS equation, applying a linearization approximation to $V_{\mathrm{xc}}$,

$$
V_{\mathrm{xc}}[n(\mathbf{r}) ; \mathbf{r}] \approx V_{\mathrm{xc}}\left[n_{\text {core }}(\mathbf{r}) ; \mathbf{r}\right]+V_{\mathrm{xc}}\left[n_{\mathrm{val}}(\mathbf{r}) ; \mathbf{r}\right]
$$

enables the definition of a new external potential, which now includes the true external potential, the Hartree potential due to the core electrons, and $V_{\text {xc }}\left[n_{\text {core }}\right]$. As a second step, this new external potential, which acts only on the valence electrons, is modified for the isolated atom such that the occupied valence wave functions are smoothened and the nodes removed. Still, the scattering properties are conserved to first order. Using such atomic pseudopotentials for ground-state calculations in poly-atomic systems typically gives reliable results. When the overlap of core and valence charge densities is large, like in alkali metals, nonlinear core corrections have to be added [14]. Then a contrived core electron density is carried along and included in the evaluation of $V_{\mathrm{xc}}$.

In the commonly employed PP approach to the $G_{0} W_{0}$ correction [Eq. (2)] the wave functions $\phi_{n \mathbf{k}}$ are replaced by $\phi_{n \mathbf{k}}^{\text {pseudo }}$, the core wave functions $\phi_{\text {core }}$ are removed, and the total electron density $n$ is replaced by $n_{\text {val }}^{\text {pseudo }}$. This implies that the core-valence xc interaction is adopted from the ( $V_{\mathrm{xc}}$-linearized) DFT scheme. This approximation is called "core-valence partitioning." To correct for this, Shirley et al. extended the PP $G W$ formalism to include core contributions to the self-energy through the incorporation of core polarization potentials into the $G W$ method [12]. The results shown for $\mathrm{Si}$ and GaAs are concomitant with the trend reported here.

In the present study we performed calculations using a newly developed $\mathrm{AE} G_{0} W_{0}$ code based on the (L)APW + lo method as implemented in the WIEN2K package [15]. To identify the influence of the approximations underlying PP results, we also carried out (L)APW + lo $G_{0} W_{0}$ calculations including only the valence wave functions and valence density in the $G_{0} W_{0}$ equations (in the following referred to as "AE-valence" calculations). In this case the correction term reads

$$
\begin{aligned}
\Delta \epsilon_{n \mathbf{k}}= & \operatorname{Re}\left(\left\langle\phi_{n \mathbf{k}}\left|\Sigma\left(\left[\left\{\phi_{n \mathbf{k}}\right\}\right], \epsilon_{n \mathbf{k}}^{\mathrm{qp}}\right)\right| \phi_{n \mathbf{k}}\right\rangle\right) \\
& -\left\langle\phi_{n \mathbf{k}}\left|V_{x c}\left[n_{\mathrm{val}}\right]\right| \phi_{n \mathbf{k}}\right\rangle .
\end{aligned}
$$

Since the wave functions and the valence density are the same, using Eq. (5) rather than Eq. (2) reveals the corevalence partitioning errors. We also performed additional PP calculations with the GWST code [16,17]. Since both PP- and AE-valence calculations apply the same "corevalence partitioning", the differences between them are only due to the softening of the PP KS wave functions in the atomic-core region.

It is well known that the $G_{0} W_{0}$ results depend on the choice of the xc functional used in the DFT calculation $[18,19]$, as is obvious for any perturbative correction. To avoid discrepancies due to different xc starting points, all ground-state calculations were performed within the LDA. We note that we do not address the issue of selfconsistency in this work $[4,19,20]$, which will exist for all the three methods, i.e., PP, AE-valence, and $\mathrm{AE} G_{0} W_{0}$, in about the same way.

All our studies were carried out at the experimental lattice constants. The integration over reciprocal space was done with a $4 \times 4 \times 4$ mesh, ensuring the convergence of the qp correction to the band gaps within $0.01 \mathrm{eV}$ for all materials except $\mathrm{C}$ and $\mathrm{GaAs}$, for which a $6 \times 6 \times 6$ mesh was needed. The frequency dependences are calculated explicitly on the imaginary axis, the self-energy is then analytically continued to the real axis. Convergence within $5 \mathrm{meV}$ with respect to the number of excited states is achieved for all the materials in both, $\mathrm{AE}$ and $\mathrm{AE}$-valence calculations. For example, in $\mathrm{Si}(\mathrm{NaCl})$ roughly 150 (250) unoccupied bands are required. For all PP- and AE-valence calculations only the outermost shell was treated in the valence region.

The LDA and qp fundamental band gaps obtained with $\mathrm{AE}$ and PP methods are shown in Table I. Our results confirm the general trend, i.e., $\mathrm{PP} G_{0} W_{0}$ calculations give in general larger band gaps than the $\mathrm{AE} G_{0} W_{0}$ method, in better agreement with experiments. The only exceptions to this rule are GaAs and CaSe. GaAs is a particular case to which we will come back later. The differences between all-electron and pseudopotential $G_{0} W_{0}$ band gaps are clearly larger than those in the LDA case. This exposes the limited reliability of the approximations implicit in the PP method for the many-body description of excited states.

In Fig. 1, we show the contribution of the pseudo-wavefunctions and core-valence partitioning to the differences between $\mathrm{AE}$ and PP $G_{0} W_{0}$ corrections to the fundamental band gaps. Two main conclusions can be drawn: Both approximations contribute to the discrepancies between

\begin{tabular}{|c|c|c|c|c|c|c|}
\hline & \multicolumn{2}{|c|}{ All-electron } & \multirow{2}{*}{$\begin{array}{c}\text { AE valence } \\
G_{0} W_{0} \\
\end{array}$} & \multicolumn{2}{|c|}{ PP } & \multirow[t]{2}{*}{ Expt. } \\
\hline & LDA & $G_{0} W_{0}$ & & LDA & $G_{0} W_{0}$ & \\
\hline $\mathrm{C}$ & 4.10 & 5.42 & 5.62 & 4.15 & 5.68 & $5.48[21]$ \\
\hline $\mathrm{Si}$ & 0.49 & 1.00 & 1.06 & 0.50 & 1.25 & $1.17[21]$ \\
\hline $\mathrm{BN}$ & 4.35 & 6.03 & 6.12 & 4.39 & 6.35 & $6.10[22]$ \\
\hline AlP & 1.44 & 2.18 & 2.21 & 1.47 & 2.50 & $2.50[21]$ \\
\hline GaAs & 0.25 & 1.29 & 0.84 & 0.35 & 0.97 & 1.63 [23] \\
\hline $\mathrm{LiF}$ & 8.97 & 13.19 & 13.32 & 8.79 & 13.56 & 14.20 [24] \\
\hline $\mathrm{NaCl}$ & 4.74 & 7.60 & 7.51 & 4.70 & 7.89 & 8.50 [25] \\
\hline $\mathrm{CaSe}$ & 1.86 & 3.36 & 3.14 & 1.84 & 3.30 & $3.85[26]$ \\
\hline
\end{tabular}

TABLE I. LDA and $G_{0} W_{0}$ fundamental band gaps (in eV) obtained with AE, AE-valence, and pseudopotential calculations. Experimental values are also provided for comparison. 


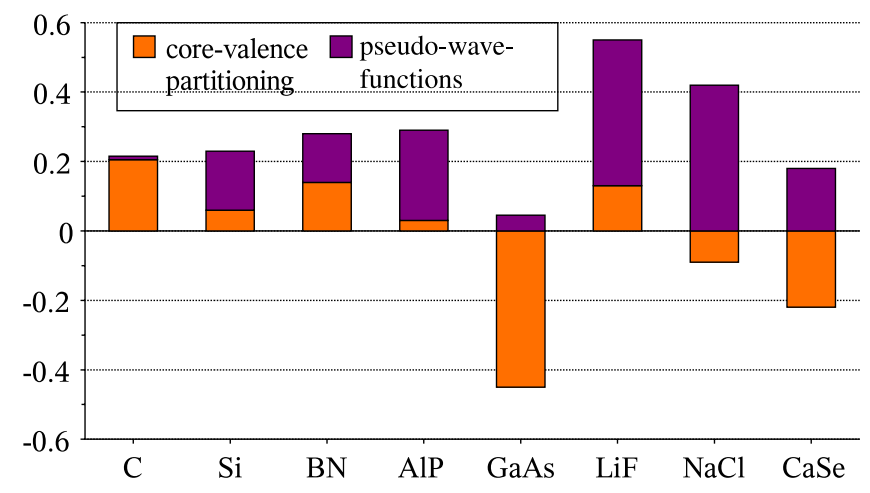

FIG. 1 (color online). Pseudo-wave-functions (black/magenta) and core-valence partitioning (gray/orange) contribution to the discrepancies between the $\mathrm{AE}$ and PP $G_{0} W_{0}$ correction to the fundamental band gap (in eV).

PP and all-electron calculations, and core-valence partitioning does not necessarily increase the $G_{0} W_{0}$ correction to the band gap. The issue of pseudo-wave-function effects has been raised previously for $\mathrm{Si}$ [4] and $\mathrm{LiF}$ [27], and its importance is now confirmed by our results.

For a detailed analysis we pick three materials, which are exemplary of the different situations we found in our studies: $\mathrm{Si}, \mathrm{NaCl}$ and $\mathrm{GaAs}$. Let us start with $\mathrm{Si}$, which historically has been the test case in this debate. The separation between core and valence electrons is clear, and PP calculations give reliable LDA results. The fundamental gap of Si is indirect, with the bottom of the conduction band lying at $85 \%$ of the distance between $\Gamma$ and $X$. For our analysis, we look at the highest occupied state at the $\Gamma$ point and the lowest unoccupied state at the $X$ point. The matrix elements of the self-energy and of the xc potential, as well as the difference between them $(\Delta F=$ $F\left(X_{c}\right)-F\left(\Gamma_{v}\right)$, where $\left.F=\Sigma_{c}, \Sigma_{x}, V_{x c}\right)$, are shown for these two states in Table II. Comparison of the AE $G_{0} W_{0}$ calculation with the AE-valence $G_{0} W_{0}$ calculations shows an increase of the $\Gamma-X$ band gap by $0.06 \mathrm{eV}$. This

TABLE II. The matrix elements of the self-energy (in eV) (correlation term: $\Sigma_{c}$, exchange term: $\Sigma_{x}$ ) and the xc potential $V_{\mathrm{xc}}$ for $\mathrm{Si}$. The top of the valence band is taken as zero for both Kohn-Sham $\left(\epsilon^{\mathrm{KS}}\right)$ and quasiparticle $\left(\epsilon^{\mathrm{qp}}\right)$ energies.

\begin{tabular}{|c|c|c|c|c|c|c|}
\hline $\mathrm{Si}$ & KS & $\Sigma_{c}$ & $\Sigma_{x}$ & $V_{\mathrm{xc}}$ & $\Sigma_{x}-V_{\mathrm{xc}}$ & $\epsilon^{\mathrm{qp}}$ \\
\hline \multicolumn{7}{|c|}{ All-electron $G_{0} W_{0}$ : } \\
\hline$\Gamma_{v}$ & 0.00 & 0.96 & -14.93 & -13.55 & -1.38 & 0.00 \\
\hline$X_{c}$ & 0.62 & -4.09 & -5.98 & -10.18 & 4.20 & 1.15 \\
\hline$X_{c}-\Gamma_{v}(\Delta)$ & 0.62 & -5.05 & 8.95 & 3.37 & 5.58 & 1.15 \\
\hline \multicolumn{7}{|c|}{ AE-valence $G_{0} W_{0}(3 s, 3 p)$} \\
\hline$\Gamma_{v}$ & 0.00 & 0.94 & -12.96 & -11.45 & -1.51 & 0.00 \\
\hline$X_{c}$ & 0.62 & -4.09 & -5.04 & -9.15 & 4.11 & 1.21 \\
\hline$X_{c}-\Gamma_{v}(\Delta)$ & 0.62 & -5.03 & 7.92 & 2.30 & 5.62 & 1.21 \\
\hline \multicolumn{7}{|c|}{$\mathrm{PP} G_{0} W_{0}$} \\
\hline$\Gamma_{v}$ & 0.00 & 0.90 & -12.92 & -11.26 & -1.64 & 0.00 \\
\hline$X_{c}$ & 0.63 & -3.96 & -5.14 & -9.09 & 3.96 & 1.38 \\
\hline$X_{c}-\Gamma_{v}(\Delta)$ & 0.63 & -4.86 & 7.78 & 2.17 & 5.60 & 1.38 \\
\hline
\end{tabular}

difference, merely due to the core-valence partitioning, can explain the mismatch between $\mathrm{AE}$ and $\mathrm{PP} G_{0} W_{0}$ $(0.23 \mathrm{eV})$ only to a small extent, in agreement with Ref. [9]. Hence, the pseudo-wave-functions play the dominant role in this example. The same is true for the fundamental band gap, as can be seen in Table I.

Analyzing the single terms of the $G_{0} W_{0}$ correction, it becomes clear that the largest differences between allelectron and PP values appear in the matrix elements corresponding to the exchange term of the self-energy $\left(\Sigma_{x}\right)$ and the $x c$ potential $\left(V_{x c}\right)$. They are as large as $2 \mathrm{eV}$. Comparing with the AE-valence values we find that these differences are almost exclusively due to the corevalence partitioning. Similar conclusions can be drawn for the contribution of these two terms to the $\Gamma-X$ gap (denoted by $\Delta \Sigma_{x}$ and $\Delta V_{x c}$ ). However, taking the difference, $\Delta \Sigma_{x}-\Delta V_{x c}$ (lines three and six in Table II), the discrepancy is reduced by almost 2 orders of magnitude $(\sim 0.04 \mathrm{eV})$. The matrix elements of the correlation term of the self-energy, $\Sigma_{c}$, in the AE-valence calculations are almost equal to the all-electron ones $(\sim 0.02 \mathrm{eV}$ difference), showing that the contribution of core electrons to $\Sigma_{c}$ is negligible. The difference in $\Delta \Sigma_{c}$ between allelectron and PP calculations $(\sim 0.2 \mathrm{eV})$, responsible for the opening of the gap, is mainly due to the pseudowave-functions.

In $\mathrm{NaCl}$, the $2 s$ and $2 p$ orbitals of $\mathrm{Na}$ are quite high in energy and play a noticeable role in the chemical bonding. It is well known that nonlinear core corrections are necessary for a reliable description of the structural properties in PP-KS calculations. Also in this case the discrepancy between $\mathrm{AE}$ and PP $G_{0} W_{0}$ band gaps is dominated by the pseudo-wave-function effect, while core-valence partitioning is smaller and counteracting. The corresponding analysis is given in Table III. At first sight the situation is similar to that observed in $\mathrm{Si}$, i.e., core-valence partitioning produces large discrepancies $(\sim 2 \mathrm{eV})$ in the $\Sigma_{x}$ and $V_{\mathrm{xc}}$ matrix elements, which are strongly reduced by considering either the difference between them or their contribution to the gap. However, in $\mathrm{NaCl}$, the omission of the semicore

TABLE III. Same as Table II for $\mathrm{NaCl}$.

\begin{tabular}{|c|c|c|c|c|c|c|}
\hline $\mathrm{NaCl}$ & $\epsilon^{\mathrm{KS}}$ & $\Sigma_{c}$ & $\Sigma_{x}$ & $V_{\mathrm{xc}}$ & $\Sigma_{x}-V_{\mathrm{xc}}$ & $\epsilon^{\mathrm{qp}}$ \\
\hline \multicolumn{7}{|c|}{ All-electron $G_{0} W_{0}$} \\
\hline$\Gamma_{v}$ & 0.00 & 2.62 & -21.07 & -16.86 & -4.21 & 0.00 \\
\hline$\Gamma_{c}$ & 4.74 & -3.31 & -5.37 & -9.95 & 4.58 & 7.60 \\
\hline$c-\Gamma_{v}(\Delta)$ & 4.74 & -5.93 & 15.70 & 6.91 & 8.79 & 7.60 \\
\hline \multicolumn{7}{|c|}{ AE-valence $G_{0} W_{0}(\mathrm{Na}: 3 s . \mathrm{Cl}: 3 s, 3 p)$} \\
\hline$\Gamma_{v}$ & 0.00 & 2.51 & -18.66 & -14.53 & -4.13 & 0.00 \\
\hline$\Gamma_{c}$ & 4.74 & -3.16 & -3.34 & -7.65 & .31 & 7.51 \\
\hline$c-\Gamma_{v}(\Delta)$ & 4.74 & -5.67 & 15.32 & 6.88 & 8.44 & 7.51 \\
\hline \multicolumn{7}{|c|}{$\mathrm{PP} G_{0} W_{0}$} \\
\hline$\Gamma_{v}$ & 0.00 & 1.99 & -18.63 & -14.22 & -4.41 & 0.00 \\
\hline & 4.70 & -3.33 & -3.51 & -7.61 & 4.10 & 7.89 \\
\hline$\Gamma_{c}-\Gamma_{v}(\Delta)$ & 4.70 & -5.32 & 15.12 & 6.61 & 8.51 & 7.89 \\
\hline
\end{tabular}


TABLE IV. Same as Table II for GaAs.

\begin{tabular}{|c|c|c|c|c|c|c|}
\hline GaAs & $\epsilon^{\mathrm{KS}}$ & $\Sigma_{c}$ & $\Sigma_{x}$ & $V_{\mathrm{xc}}$ & $\Sigma_{x}-V_{\mathrm{xc}}$ & $\epsilon^{\mathrm{qp}}$ \\
\hline \multicolumn{7}{|c|}{ All-electron $G_{0} W_{0}$ : } \\
\hline$\Gamma_{v}$ & 0.00 & 1.28 & -16.85 & -15.64 & -1.21 & 0.00 \\
\hline$\Gamma_{c}$ & 0.25 & -3.34 & -12.22 & -16.67 & 4.45 & 1.29 \\
\hline$\Gamma_{c}-\Gamma_{v}(\Delta)$ & 0.25 & -4.62 & 4.63 & -1.03 & 5.66 & 1.29 \\
\hline \multicolumn{7}{|c|}{ AE-valence $G_{0} W_{0}(\mathrm{Ga}: 4 s, 4 p$. As: $4 s, 4 p)$} \\
\hline$\Gamma_{v}$ & 0.00 & 1.00 & -12.39 & -11.38 & -1.01 & 0.00 \\
\hline$\Gamma_{c}$ & 0.25 & -3.17 & -6.88 & -10.63 & 3.75 & 0.84 \\
\hline$\Gamma_{c}-\Gamma_{v}(\Delta)$ & 0.25 & -4.17 & 5.51 & 0.75 & 4.76 & 0.84 \\
\hline \multicolumn{7}{|c|}{$\mathrm{PP} G_{0} W_{0}$} \\
\hline$\Gamma_{v}$ & 0.00 & 0.82 & -12.65 & -11.24 & -1.41 & 0.00 \\
\hline$\Gamma_{c}$ & 0.35 & -3.29 & -7.01 & -10.33 & 3.32 & 0.97 \\
\hline$\Gamma_{c}-\Gamma_{v}(\Delta)$ & 0.35 & -4.11 & 5.64 & 0.91 & 4.73 & 0.97 \\
\hline
\end{tabular}

states produces noticeable changes, both in $\Delta \Sigma_{c}$ and $\Delta \Sigma_{x}-\Delta V_{x c}(\sim 0.3 \mathrm{eV})$ which tend to cancel each other. Comparing AE-valence and PP-matrix elements, we observe that the pseudo-wave-function effects on $\Sigma_{x}$ and $V_{\text {xc }}$ compensate each other almost completely. However, the additional reduction of the correlation term results in an increase of the band gap correction.

GaAs is special in the sense that the $3 d$ levels of $\mathrm{Ga}$ are close to the $4 s$ levels of As and hence play an important role in the bonding. Contrary to the previous cases, corevalence partitioning clearly leads to a sizeable difference in the band gap, reducing it by around $0.5 \mathrm{eV}(\sim 30 \%)$. This discrepancy is only slightly compensated by the effect of the pseudo-wave-functions. The matrix elements for the highest occupied and lowest unoccupied states at the $\Gamma$ point are shown in Table IV. Again, the largest discrepancies, up to $6 \mathrm{eV}$, appear in the $\Sigma_{x}$ and $V_{\mathrm{xc}}$ matrix elements and originate from core-valence partitioning. Looking at either the difference between these two terms or their contribution to the band gap, we again find pronounced cancellation effects. However, due to the large differences in the single contributions, a sizeable discrepancy of almost $1 \mathrm{eV}$ in $\Delta \Sigma_{x}-\Delta V_{\mathrm{xc}}$ remains. This is not fully compensated by the diminution of the correlation term $(0.5 \mathrm{eV})$. The same trend has been observed recently in PAW calculations [9]. Although PP- $G_{0} W_{0}$ values are similar to the AE-valence ones, small deviations due to the pseudo-wave-functions are obtained in the band gap correction.

Summarizing, both core-valence partitioning and pseudo-wave-functions are equally important sources of inaccuracy in PP $G_{0} W_{0}$ band gaps. The former produces differences of several eV's in the $\Sigma_{x}$ and $V_{\mathrm{xc}}$ matrix elements. However, these tend to cancel each other, such that the remaining discrepancy may even be much smaller than that originating from the pseudo-wave-functions, as is the case for $\mathrm{Si}, \mathrm{AlP}, \mathrm{LiF}$, and $\mathrm{NaCl}$. The latter, although small in the matrix elements, plays a major role in the opening of band gaps, mainly through the underestimation of the correlation contribution. In contrast to the common belief that the inclusion of core states would result in reduced band gaps, the AE-valence results for $\mathrm{NaCl}, \mathrm{GaAs}$, and CaSe demonstrate that this is not a systematic trend. Furthermore, GaAs and CaSe exhibit even smaller band gaps whithin PP than in the AE treatment. In GaAs, omission of the third shell in the calculation of the self-energy makes core-valence partitioning inappropriate. We conclude that, in order to evaluate the $G_{0} W_{0}$ approximation, a full-potential all-electron treatment is required.

We acknowledge C. Freysoldt, P. Rinke and H. Jiang for useful discussions, and the Nanoquanta $\mathrm{NoE}$ and the Austrian Science Fund for their financial support.

[1] L. Hedin, Phys. Rev. 139, A796 (1965).

[2] M. S. Hybertsen and S. G. Louie, Phys. Rev. Lett. 55, 1418 (1985).

[3] F. Aryasetiawan and O. Gunnarsson, Rep. Prog. Phys. 61, 237 (1998).

[4] W. Ku and A. G. Eguiluz, Phys. Rev. Lett. 89, 126401 (2002).

[5] K. Delaney et al., Phys. Rev. Lett. 93, 249701 (2004).

[6] M. L. Tiago, S. Ismail-Beigi, and S. G. Louie, Phys. Rev. B 69, 125212 (2004).

[7] M. van Schilfgaarde, T. Kotani, and S. V. Faleev, Phys. Rev. B 74, 245125 (2006).

[8] C. Friedrich et al., Phys. Rev. B 74, 045104 (2006).

[9] M. Shishkin and G. Kresse, Phys. Rev. B 74, 035101 (2006).

[10] R. W. Godby et al., Phys. Rev. B 36, 6497 (1987).

[11] A. Fleszar and W. Hanke, Phys. Rev. B 56, 10228 (1997).

[12] E. L. Shirley, X. Zhu, and S. G. Louie, Phys. Rev. B 56, 6648 (1997).

[13] F. Gygi and A. Baldereschi, Phys. Rev. Lett. 62, 2160 (1989).

[14] S. G. Louie, S. Froyen, and M. L. Cohen, Phys. Rev. B 26, 1738 (1982).

[15] P. Blaha et al., WIEN2k, An Augmented Plane Wave Plus Local Orbitals Program for Calculating Crystal Properties (Austria, 2002).

[16] M. M. Rieger et al., Comput. Phys. Commun. 117, 211 (1999).

[17] L. Steinbeck et al., Comput. Phys. Commun. 125, 105 (2000).

[18] P. Rinke et al., New J. Phys. 7, 126 (2005).

[19] F. Bruneval, N. Vast, and L. Reining, Phys. Rev. B 74, 045102 (2006).

[20] M. van Schilfgaarde, T. Kotani, and S. Faleev, Phys. Rev. Lett. 96, 226402 (2006).

[21] O. Madelung, M. Schulz, and H. Weiss, Intrinsic Properties of Group IV Elements and III-V, II-VI and I-VII Compounds, Landolt-Börnstein, New Series, Group III/22A (Springer-Verlag, New York, 1987).

[22] N. Miyata et al., Phys. Rev. B 40, 12028 (1989).

[23] D. E. Aspnes, Phys. Rev. B 14, 5331 (1976).

[24] M. Piacentini, D. W. Lynch, and C. G. Olson, Phys. Rev. B 13, 5530 (1976).

[25] R. T. Poole et al., Phys. Rev. B 11, 5179 (1975).

[26] Y. Kaneko and T. Koda, J. Cryst. Growth 86, 72 (1990).

[27] P. Puschnig and C. Ambrosch-Draxl, Phys. Rev. B 66, 165105 (2002). 